\title{
Vigor Resourceful Practice for Two-Way Networks
}

\author{
N. Sunil Kumar ${ }^{1}$, B.Naveen Kumar ${ }^{2}$, S.Bala Naresh ${ }^{3}$, P.Narahari ${ }^{4}$ \\ 1, 3, 4. M.Tech students, 2. Assistant Professor \\ Global College of Engineering \& Technology, kadapa.
}

\begin{abstract}
In this paper, we present a new medium access control (MAC) protocol forad-hoc networks with multiple input multiple output (MIMO) links. Instead of using perfect synchronizationtechnique, we assume the cooperative transmission is asynchronous. It is shown that the energy savings of 39\% and 56\% areachievable in line and grid networks with a large number of nodes, respectively.This paper describes theassumptions, the implementation process and the challenges wewere presented with. The MAC protocol proposed in addresses thisissue using an efficient cooperative scheme. Ageneral decision feedback equalizer (DFE) is used in the receiving cluster members to equalize the received MISO signal and detect as soft symbols. The receiving cluster members send the soft decision outputs to the destination node.Up to $80 \%$ in energysavings can be achieved for a grid topology, while for random nodeplacement our cooperative protocol can save up to $40 \%$ in energyconsumption relative to the other protocols.
\end{abstract}

\section{INTRODUCTION}

In many applications, the nodes are small and havelimited and nonreplenishable energy supplies. For this reason,energy conservation is critical for extending the lifetime of thesenetworks, and it is not surprising that the problem of energy efficiencyand energy-efficient communication in ad hoc networkshas received a lot of attention in the past several years. Thisproblem, however, can be approached from two different angles:

Energy-efficient route selection algorithms at the networklayer or efficient communication schemes at the physical layer. In cooperativetransmission, multiple nodes simultaneously receive, decode, and retransmit data packets. In this paper, as opposed to previousworks, we use a cooperative communication model withmultiple nodes on both ends of a hop and with each data packetbeing transmitted only once per hop.

Exchange of data-ACK frames for Cooperative MAC

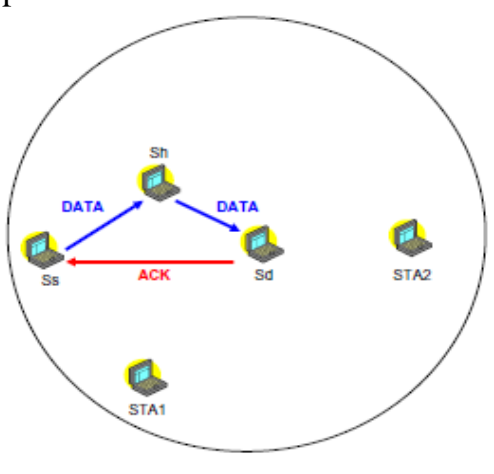

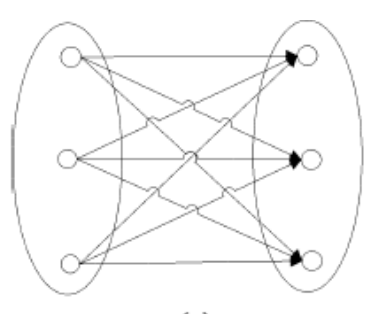

(a)

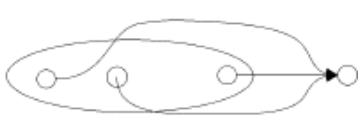

(b)

Fig. 2. (a) Our cooperative reception model and (b) the CAN reception model.

Our model of cooperative transmission for a single hop isfurther depicted in Fig. 2(a). Wireless networks that provide multi-rate support give thestations the ability to adapt their transmission rate to the link quality in order to make their transmissions more reliable.Thus, stations that experience poor channel conditions tend touse lower transmission rates and vice versa 
Our cooperative transmission protocol consists of two phases.In the routing phase, the initial path between the source andthe sink nodes is discovered as an underlying "one-node-thick"path. Then, the path undergoes a thickening process in the "recruiting-and-transmitting" phase. In this phase, the nodeson the initial path become cluster heads, which recruit additionaladjacent nodes from their neighborhood.

Instead of having a slow stationtransmitting its frame directly to the Access Point, an alternativeroute through a high speed station is used sending theframe in a two-hop manner. We implemented this protocol by modifying the Linuxwireless driver HostAP.The presence of multiple elements at both ends of the link creates independent channels in the presence of multipath or rich scattering. Multiple independent data streams can be transmitted simultaneously on these different channels to provide extremely high spectral efficiencies (increase in capacity) that comes at the cost of no extra bandwidth or power

In cooperative networks, the transmitting nodes use idle nodes as relays to reduce the adverse effect of multi-path fading in wireless channels. Different cooperative schemes and performance evaluation are discussed. An overview of cooperative transmission systems is given in and the performance of several cooperation methods such as amplify-and forward cooperation, decode-and-forward cooperation, and coded cooperation are evaluated.

\section{COOPERATIVE PROTOCOL}

When the source node wants to transmit information tothe destination node, both source node and destination node recruit neighbor nodes and form the transmitting and receiving cluster respectively. The source node and destination node areautomatically the master nodes in their respective clusters. Thesource node then transmits information to its cluster membersand destination.For the purpose ofperformance evaluation, we chose to implement this phaseusing the Ad hoc on-demand Distance-Vector routing protocol (AODV).The main novelty of our paper - the "recruiting-and-transmitting"phase - is done dynamically per hop, starting fromthe source node and progressing, hop by hop, as the packetmoves along the path to the sink node.

\section{A. Operation of the "Recruit-and-Transmit" Phase}

The main novelty of our paper - the "recruiting-and-transmitting"phase - is done dynamically per hop, starting fromthe source node and progressing, hop by hop, as the packetmoves along the path to the sink node.
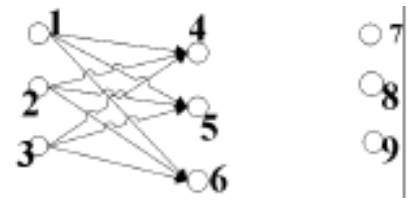

In the current hop,node 2 is the sending cluster head and has a packet to be sentto node 5 . Node 2 sends a request-to-recruit (RR) packet to node 5 , causing node 5 to start the formation of thereceiving cluster, with node 5 as the cluster head. From the routing phase, node 5 knows that the next-hop node is node 8 . Node 5 broadcasts to its neighbors a recruit (REC) packet. The REC packet contains: the id of the previous node, the id of the next node, and the maximum timeto respond. A potential recruit replies to the REC packet with a grant (GR) packet that contains the computed sum after a random back off time drawn uniformly from $(0, T)$. The GR packets inform the cluster head that the nodes are available to cooperate in receiving on the current hop and in sending on the next hop.

\section{B. Details of the Control Packets}

The format of an RRpacket includes: the id of the sender node, the id of the receiver node, the sink node id, and the NAV field that containsthe estimated transmission time of the data packet. The NAVfield serves to indicate when the channel will become availableagain for other transmissions. The REC packet contains thesender node id, the receiver node id, the id of the next node on the path, and the maximum time-torespond. The GR packet sent from node contains the id of theoriginator of the REC packet and the sum of the link costs of thereceiving link and the sending link. A node can be involved in asingle recruiting process at any time; i.e., a node can have onlyone outstanding GR packet. A node chosen to cooperate cannotbe involved in another recruiting process until the transmissionof the current data packet is fully completed, i.e., received andsent to the next cluster by the cooperating node.

\section{Assumptions of the Cooperative Models}

We calculate the probability of error of the cooperative protocolbased on a formula derived in the Appendix. Our model ofcooperative communication assumes $m$ transmitters located inthe sending cluster and a single receiver located in the receiving 
cluster. In this sense, the model is similar to the MISO case. Notethat each receiver in the receiving cluster creates an independent Orthogonal system, which could be implemented throughtime-, frequency-, or codeorthogonality. In particular, we assume the MISO case of $m$ transmitters overRayleigh-fadedchannels with known channel state information (CSI).

\section{ENERGY CONSUMPTION ANALYSIS}

In this section we would like to consider the capacity inproposed cooperative MIMO system.We compare the energy consumption of our cooperative protocolto the CAN protocol and the disjoint-paths scheme.To make the comparison of energy consumption of anytwo schemes meaningful, the failure probability, as defined in protocol robustness, needs to be kept equal for the compared schemes. When $m$ is small, regardless of, there is energy saving forour cooperative protocol over the disjoint-paths scheme. When mis larger than 3 , a value of $\beta \geq 2$ achieves energy savings.Consequently, when the distance between the sending and thereceiving clusters is small, one should use a small number ofcooperative nodes, such as. When this distance is large,one should use larger. Our cooperative protocol can save up to60\% in energy over the disjoint-paths scheme and up to $80 \%$ in energy over the CAN protocol for large values of. The amountof savings increases as the failure probability decreases and asincreases.

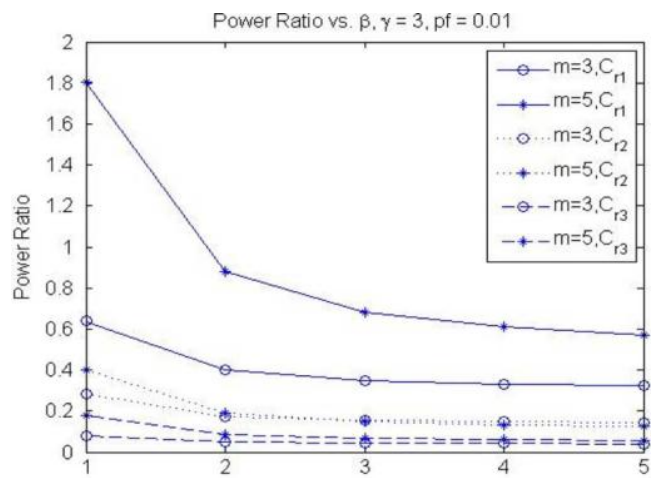

\section{EXPERIMENTS}

In our experiments, we use the basic setup of three stations,with a source, a destination, and a helper. We ran differentexperiments changing the position of the helper between thedifferent regions. The first experiment is the comparison between the twoprotocol schemes we described in the previous section: theunicast transmissions scheme and the broadcast transmissionsscheme. We expected to have higher throughput in the case ofbroadcast transmissions as in this case we did not have theadded overhead from the ACK transmission. Studying the TCP window size in eachexperiment, we concluded that this is due to the fact that in thebroadcast transmissions scheme, there is no acknowledgmentfor the MAC transmissions. Our next experiment was to study the overhead that isadded by the modifications we made to the HostAP driver.Even though it is reasonable to assume that the processingtime introduced by the addition of the Coop Header in thesource and by the examination of this header at the destination. Will have a negligible effect on overall transmission times, we decided to verify that assumption by comparing the totalfile transfer time between two stations with and without our modifications. This experiment was conducted with directtransmissions from the source to destination.

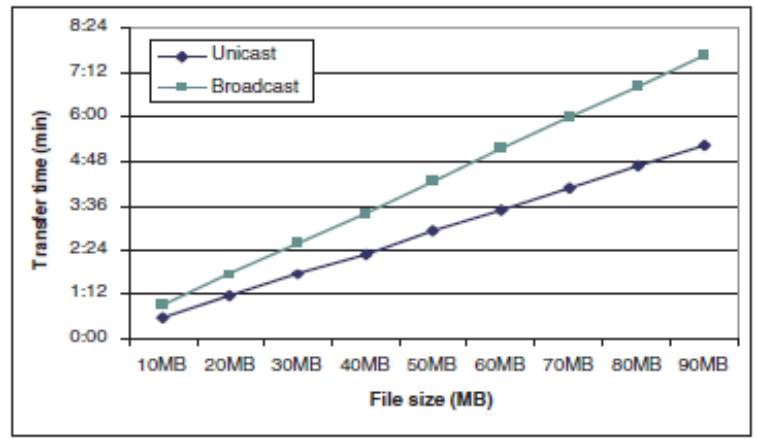

V.

SYSTEM CAPACITY ANALYSIS

From simulation result, the performance of proposed cooperativeMIMO scheme is close to MIMO system with corresponding number of antennas. However, cooperative MIMO scheme needs to deal with intra 
cluster transmission in both source cluster and destination cluster. Although cooperative MIMO scheme provides spatial diversity, the transmission capacity decreases due to node cooperation. In this section we would like to consider the capacity in proposed cooperative MIMO system. We start the analysis with a simple model, where only one transmitting cluster and one receiving cluster exist. The system assumption is as follows:

1) Only one transmitting cluster and one receiving cluster. We do not consider interference and opportunity cost here.

2) The size of transmitting cluster is $\mathrm{M}+1$ and the size of receiving cluster is $\mathrm{N}+1$.

3) The radius in cluster recruiting algorithm is $\mathrm{r}$.

4) Signals degrade due to Path loss. The path loss constant $\alpha$ is usually between 2 and 4 .

\section{II. MIMO BACKGROUND}

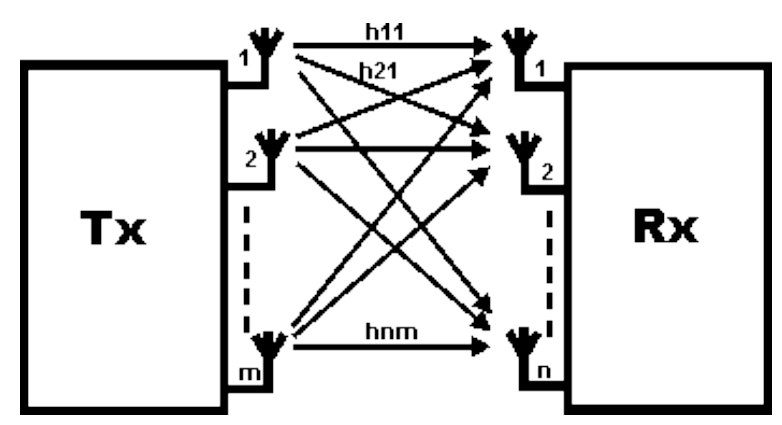

A MIMO link employs MEAs at both the transmitter ( $\mathrm{M}$ elements)and the receiver ( $\mathrm{N}$ elements) as shown in Figure 1. The presence of multiple elements at both ends of the communication link opens up independent channels (streams) for transmission in the presence of multipath or rich scattering. A transmitter has one of two options: it can either send dependent (correlated) signals on the different antenna elements or send independent signals.

MIMO technology takes advantage of a radio-wave phenomenon called multipath where transmitted information bounces off walls, ceilings, and other objects, reaching the receiving antenna multiple times via different angles and at slightly different times. Multipath is a natural occurrence for all radio sources. Radio signals bounce off objects and move at different speeds towards the receiver. In the past multipath caused interference and slowed down wireless signals. MIMO takes advantage of multipath to combine the information from multiple signals improving both speed and data integrity.

\section{CONCLUSION}

We propose a new method for asynchronous cooperativeMIMO communication. We have identified the potential advantages of MIMO links in wireless ad-hoc networks. The problem of fair channel allocation for the target environment has been presented and the key optimization considerations for the design of an ideal MAC protocol for such an environment have been discussed. The cooperative scheme takes advantage of the fact that the helper is selected in a way such that the two links (source to helper and helper to destination) have high transmission rates. We proposed an energy-efficient cooperative protocol, and we analyzed the robustness of the protocol to data packet loss. We used DP to formulate the optimal cooperative routing problem as a multistage decision problem. The problem of finding the optimal route was shown to be equivalent to finding the shortest path in the corresponding cooperation graph. The total energy consumption was analytically computed, illustrating substantial energy savings. For example, when nodes are positioned on a grid, the energy savings of our cooperative protocol over the CAN protocol is up to $80 \%$.

\section{REFERENCES}

[1] A. Khandani, J. Abounadi, E. Modiano, and L. Zheng, "Cooperative routing in static wireless networks," IEEE Trans. Commun., vol. 55, no. 11, pp. 2185-2192, Nov. 2007.

[2] C. Chou, J. Yang, and D. Wang, "Cooperative MAC protocol with automaticrelay selection in distributed wireless networks," in Proc. IEEE Int. Conf. Pervasive Comput. Commun. Workshops, White Plains, NY, Mar. 2007, pp. 526-531.

[3] T. Korakis, S. Narayanan, A. Bagri, and S. Panwar, "Implementing a cooperative MAC protocol for wireless LANs," in Proc. IEEE ICC, Istanbul, Turkey, Jun. 2006, vol. 10, pp. 4805-4810.

[4] H. Shen and S. Kalyanaraman, "Asynchronous cooperative MIMO communication," in Proc. IEEE WiOpt, Limassol, Cyprus, Apr. 2007, pp. 1-9.

[5] K. Sundaresan, R. Sivakumar, M. Ingram, and T. Chang, "A fair medium access control protocol for ad-hoc networks with MIMO links," in Proc. IEEE INFOCOM, Hong Kong, Mar. 2004, vol. 4, pp. 2559-2570. 